\title{
COMMON SECANTS FOR PLANE CONVEX SETS ${ }^{1}$
}

\author{
V. L. KLEE, JR.
}

A well known theorem of Helly [1] asserts that if $\mathfrak{F}$ is a finite family of convex subsets of $E^{n}$ in which each $n+1$ members have a common point, then there is a point common to all members of $\mathfrak{F}$. In this connection, Vincensini $[5 ; 6]$ has raised the following question: For $n \geqq 2$ and $0 \leqq r \leqq n-1$, is there an integer $k=k(n, r)$ such that if $\mathfrak{F}$ is an arbitrary finite family of convex subsets of $E^{n}$ in which each $k$ members have a common $r$-secant (i.e., an $r$-dimensional linear variety intersecting all $k$ of them), then there is an $r$-secant common to all members of $\mathfrak{F}$ ? Helly's theorem shows that for $r=0$ the answer is affirmative, with $k(n, 0)=n+1$. But for $0<r \leqq n-1$ the answer is always negative, for Santaló [3] has remarked that for each $k \geqq 2$ there are $k+1$ line segments in $E^{2}$ which have no common 1-secant even though each $k$ of them do have a common 1-secant; from this it follows easily that the assertion is true with 2 and 1 replaced by $m$ and $m-1$ respectively, and hence that no $k(n, r)$ having the required properties can exist for $0<r \leqq n-1$. (Santaló's result, stated in [3] without proof, is not difficult to prove.)

It is still of interest to determine whether the desired $k(n, r)$ may exist under additional restrictions on the family $\mathfrak{F}$, and for $n=2$ the following is known (where "secant" henceforth means "1-secant")Suppose $\mathfrak{F}$ is a finite family of plane convex sets, each $k$ of which have a common secant. Then each of the following conditions assures the existence of a secant common to all members of $\mathfrak{F}$ : (S) $k=3$, and the members of $\mathfrak{F}$ are line segments, all of which are parallel; (V) $k=4$, and there is a line in the plane none of whose parallels intersects more than one member of $\mathfrak{F}$. ((S) has been proved by Santalo [4] and by Rademacher and Schoenberg [2]. (V) is given by Vincensini [6]. Still other conditions have been given by Santalo $[3 ; 4]$.) The purpose of this note is to show that (V) remains sufficient when " $k=4$ " is replaced by " $k=3$." The resulting theorem includes both of those just mentioned.

THEOREM. Suppose $\mathfrak{F}$ is a family of connected plane sets, either $\mathfrak{F}$ is finite or each member of $\mathfrak{F}$ is compact, and there is a line in the plane none of whose parallels intersects more than one member of $\mathfrak{F}$. Then if each 3 members of $\mathfrak{F}$ have a common secant, there is a secant common to all members of $\mathfrak{F}$.

Received by the editors November 23, 1953.

${ }^{1}$ Sponsored by the Office of Ordnance Research, U.S. Army. 
Proof. We first describe some more or less obvious reductions of the problem. Since the convex hull of a compact plane set is compact, and since (as was remarked by Vincensini [6]) a connected plane set is intersected by a given line in the plane if and only if its convex hull is intersected by the line, we may assume that all members of $\mathfrak{F}$ are convex. The usual sort of argument shows that the result for the compact case follows from that for the finite case, so we may assume also that $\mathfrak{F}$ is finite. Now for each triple of sets of $\mathfrak{F}$ we may choose three collinear points (not necessarily distinct), one from each set. Let $P$ be the set of all points so chosen and for each $F \in \mathfrak{F}$ let $F^{1}$ be the convex hull of $F \cap P$. To prove the theorem for $\mathfrak{F}$ it suffices to do so for $\left\{F^{1}: F \in \mathfrak{F}\right\}$. Thus the proof will be complete if we can prove the following: Suppose $k \geqq 3$ and $B$ is a set of $k+1$ convex polygons in the plane, each $k$ of which have a common secant. (Some members of (3) may be degenerate.) Suppose there is a line $L$ in the plane none of whose parallels intersects more than one member of $(\mathfrak{S}$. Then there is a secant common to all members of $(\$)$.

For each $G \in \mathcal{B}$, let $p_{G}$ be the centroid of $G$, and for each $t \geqq 0$ let $G_{t}$ be the set obtained by expanding $G$ in the ratio $t$ about $p_{G}$ (i.e., $\left.G_{t}=(1-t) p_{G}+t G\right)$. An easy compactness argument shows that there is a least value $s \geqq 0$ of $t$ for which each $k$ members of $\left\{G_{t}: G \in(S)\right.$ have a common secant. Let $\mathfrak{S}=\left\{G_{s}: G \in(S)\right.$. Clearly $s \leqq 1$, so always $G_{s} \subset G$ and our proof can be completed by showing that there is a secant common to all members of $\mathfrak{S}$. From the minimal nature of $s$ it follows that either $s=0$ (in which case the desired conclusion is obvious) or there are $k$ sets of $\mathfrak{S}$ such that every line which intersects all of them must properly bound (i.e., bound but not contain) at least one of them. By an appropriate affine transformation of the plane onto itself, we may assume that the $x$-axis intersects each of these $k$ sets and that no line parallel to the $y$-axis intersects more than one member of $\mathfrak{S}$. For arbitrary members $U$ and $V$ of $\mathfrak{S}$, write $U<V$ provided $u<v$ whenever these are the $x$-coördinates of points of $U$ and $V$ respectively. Then $\mathfrak{S}$ is linearly ordered by the relation $<$, and we may name the $k$ sets under consideration in such a way that $H_{1}$ $<H_{2}<\cdots<H_{k}$. Let $I$ be the remaining member of $\mathfrak{S}$. If the $x$-axis intersects $H$ also, then it is a secant common to all members of $\mathfrak{S}$ and the proof is complete, so suppose this is not the case. By using this supposition, we shall show in the next paragraph that at least one of the sets $H_{i}$ is merely a point, and this fact will be used to complete the proof in the final paragraph.

Suppose each $H_{i}$ includes at least two points. A plane set will be called an $A$-set [a $B$-set] provided it includes at least one point hav- 
ing positive [negative] $y$-coördinates. Since each of the sets $H_{i}$ is convex, includes at least two points, and intersects the $x$-axis, it is pictorially evident (and can be rigorously proved) that if there is a $j$ with $1 \leqq j \leqq k$ such that $H_{i}$ is an $A$-set whenever $1 \leqq i<j$ and a $B$-set whenever $j<i \leqq k$, or vice versa, then there is a line which intersects each of the $H_{i}$ 's but bounds none of them. Since this is impossible, it follows that there are three members, $J, K$, and $L$ of $\left\{H_{i}: 1 \leqq i \leqq k\right\}$, such that $J<K<L$ and either $J$ is not a $B$-set, $K$ is not an $A$-set, and $L$ is not a $B$-set, or the same thing with the $A$ 's and $B$ 's interchanged. We lose no generality by considering only the first possibility. Now it can be seen that for each possible position of the set $H$ (knowing that $H$ does not intersect the $x$-axis and that $\mathfrak{S}$ is linearly ordered by $<)$, there are two sets from $\{J, K, L\}$ which have no secant in common with $H$. (For example, if $H$ is an $A$-set and $H<K$, then $H, K$, and $L$ have no common secant; if $H$ is a $B$-set and $H<J$, then $H, J$, and $K$ have none; if $H$ is a $B$-set and $J<H<K$, then $J, H$, and $L$ have none.) Since this contradicts the fact that each $k$ members of $\mathfrak{S}$ have a common secant (with $k \geqq 3$ ), we conclude that some member of $\mathfrak{S}$ is merely a point (which we assume to be the origin $\phi)$, and will complete the proof from this.

For each $H \in \mathfrak{S}$ let $m H$ be the set of all numbers $t$ such that the line $y=t x$ intersects $H$. Since $H$ is convex and either $H<\{\phi\}$ or $\{\phi\}<H, m H$ is a convex set of real numbers. Since $\{\phi\} \in \mathfrak{S}$ and each three members of $\mathfrak{S}$ have a common secant, each two members of $\{m H: H \in \mathfrak{S}\}$ must have a common point. But then by Helly's theorem in one dimension (which is very easy to prove) there is a number $r$ in common to all the sets $m H$, and the line $y=r x$ is a secant common to all members of $\mathfrak{S}$. This concludes the proof and the paper.

\section{REFERENCES}

1. E. Helly, Über Mengen konvexer Körper mit gemeinschaftlichen Punkten, Jber. Deutschen Math. Verein vol. 32 (1923) pp. 175-176.

2. Hans Rademacher and I. J. Schoenberg, Convex domains and Tchebycheff's approximation problem, Canadian Journal of Mathematics vol. 2 (1950) pp. 245-256.

3. L. A. Santal6, A theorem on sets of parallelepipeds with parallel edges, Publ. Inst. Mat. Univ. Nac. Litoral vol. 2 (1940) pp. 49-60.

4. - Supplement to the note: A theorem on sets of parallelepipeds with parallel edges, ibid. vol. 3 (1942) pp. 202-210.

5. P. Vincensini, Figures convexes et variêtés linéaires de l'espace euclidien à $n$ dimensions, Bull. Sciences Math. (2) vol. 59 (1935) pp. 163-174.

6. - Les ensembles d'arcs d'un même cercle dans leurs relations avec les ensembles de corps connexes du plan euclidien, Atti del Quarto Congresso dell'Unione Matematica Italiana, Taormina, 1951, vol. II, pp. 454-464. Rome, Perella, 1953.

UNIVERSITY OF WASHINGTON 